\title{
Sequent Calculi for Branching Time Temporal Logics of Knowledge and Belief
}

\author{
Jūratè SAKALAUSKAITE் \\ Institute of Mathematics and Informatics \\ Akademijos 4, 08663 Vilnius, Lithuania \\ e-mail: jurates@ktl.mii.lt
}

Received: April 2006

\begin{abstract}
In this paper we consider branching time temporal logics of knowledge and belief. These logics involve the discrete time linear temporal logic operators "next" and "until" with the branching temporal logic operator "on all paths". The latter operator is interpreted with respect to a version of the bundle semantics. In addition the temporal logic of knowledge (belief) contains an indexed set of unary modal operators "agent i knows" ("agent i believes") and it contains the modality of common knowledge (belief). For these logics we present sequent calculi with a restricted cut rule. Thus, we get proof systems where proof-search becomes decidable. The soundness and completeness for these calculi are proved.
\end{abstract}

Key words: temporal logics of knowledge and belief, branching time, sequent calculus, restricted cut rule.

\section{Introduction}

Temporal logics of knowledge and belief are becoming increasingly important in both mainstream computer science and AI. In AI, temporal logics of knowledge and belief are used as knowledge representation formalism (Catach, 1988), and may be used in the specification and verification of distributed intelligent systems (Wooldridge, 1992).

In this paper we consider the branching time temporal logics of knowledge and belief. These logics involve the discrete time linear temporal logic operators "next" and "until" with the branching temporal logic operator "on all paths". The latter operator is interpreted with respect to a version of the bundle semantics (Stirling, 1992). In addition the temporal logic of knowledge (belief) contains an indexed set of unary modal operators "agent i knows" ("agent i believes") that allow to represent the information possessed by the group of agents. These operators satisfy the analogues of the modal logic $S 5$ (KD45).This system is widely accepted as a logic of idealized knowledge (belief) (Fagin et al., 1995; Halpern and Moses, 1992). Also this logic contains the modality of common knowledge (belief). For these logics we present sequent calculi with a restricted cut rule. Thus, we get proof systems where proof-search becomes decidable. The soundness and completeness for these calculi are proved. Our work uses the ideas from Alberucci (2002); Halpern et al. (2004) and van der Meyden et al. (2003). 
We mention some related works. In van der Meyden et al. (2003) the Hilbert style axiom system is presented for branching time temporal logic of knowledge. The linear time temporal logic of knowledge without common knowledge operator is considered in (Wooldridge et al., 1998; Dixon et al., 1998). In Wooldridge et al. (1998) a tableau based decision procedure is presented for the considered logic. In Dixon et al. (1998) a resolution-based proof system is presented which is shown to be correct. The logic of common knowledge without temporal operators is considered in Alberucci (2002), where complete Tait-style sequent calculus with restricted cut rule for the logic is presented.

The paper is organized as follows. In the next section we provide formal definitions for the logics we consider. In Section 3 we present sequent calculi and prove the soundness theorem. In Section 4 we prove the completeness of the presented sequent calculi. In Section 5 we present an algorithm to check the provability in the considered sequent calculi.

\section{Language and Semantics}

To define the language $\mathcal{L}$ for the temporal logic of knowledge (belief) we start from a set of primitive propositions $P=\{p, q, \ldots\}$, the constant true, the propositional connectives $\neg, \wedge, \vee$, the epistemic modalities $[1], \ldots,[n]$, the epistemic modality $E$, the common knowledge (belief) modality $C$ and the temporal modalities: unary operator $\circ$, a binary operator $U$ and unary operator $A$. If $\phi$ is a formula $[i] \phi$ says that agent $i$ knows (believes) $\phi$, a formula $E \phi$ says that every agent knows (believes) $\phi$, a formula $C \phi$ says that $\phi$ is a common knowledge (belief) of all agents, a formula $\circ \phi$ says that $\phi$ is true at the next time moment, a formula $\phi U \psi$ says that $\phi$ holds until $\psi$ does, a formula $A \phi$ says that on all paths $\phi$ holds.

The semantics for all operators except for the operator $A$ is defined as in Wooldridge $e t$ al. (1998). The semantics for $A$ is defined as in Stirling (1992), which is called the bundle semantics. We assume that the world may be in any of a set $S$ of states. We generally use $s$ to denote a state. The internal structure of states is not an issue in this work. A timeline $l$ is an infinitely long, linear, discrete sequence of states, indexed by natural numbers. For convenience, we define a timeline $l$ to be a total function $l: N \rightarrow S$. Let Tlines be the set of all timelines. Note that timelines correspond to the runs of Halpern, Meyden and Vardi (Halpern et al., 2004). A point, $p$, is pair $(l, u)$, where $l \in$ Tlines is a timeline and $u \in N$ is a temporal index into $l$. Any point $(l, u)$ will uniquely identify a state $l(u)$. Two timelines $l, l^{\prime}$ are equivalent to time $n$ if $l(j)=l^{\prime}(j)$ for $j=0, \ldots, n$. Let the set of all points (over $S$ ) be Points. We then let an agent's knowledge (belief) accessibility relation $R_{i}$ hold over Points, i.e., $R_{i} \subseteq$ Points $\times$ Points, for all $i \in\{1, \ldots, n\}$. A valuation, $\pi$, is a function $\pi$ : Points $\times P \rightarrow\{T, F\}$. We can now define models for $\mathcal{L}$.

A model, $M$, for $\mathcal{L}$, is a structure $M=\left(T L, R_{1}, \ldots, R_{n}, \pi\right)$, where:

- $T L \subseteq$ Tlines is a set of timelines;

- $R_{i}$, for all $i \in\{1, \ldots, n\}$, is an agent accessibility relation over Points, i.e., $R_{i} \subseteq$ Points $\times$ Points;

- $\pi$ : Points $\times P \rightarrow\{T, F\}$ is a valuation. 
As usual, we define the semantics of the language via satisfaction relation " $=$ ". For $\mathcal{L}$, this relation holds between pairs of the form $(M,(l, u))$, where $M$ is a model and $(l, u)$ is a point of $M$, and $\mathcal{L}$ formulas. $E \phi$ stands for $[1] \phi \wedge \ldots \wedge[n] \phi$.

- $(M,(l, u)) \models$ true;

- $(M,(l, u)) \models p$ iff $\pi(l, u)(p)=T$ (where $p \in P$ );

- $(M,(l, u)) \models \neg \phi$ iff $(M,(l, u)) \not=\phi$;

- $(M,(l, u)) \models \phi \vee \psi$ iff $(M,(l, u)) \models \phi$ or $(M,(l, u)) \models \psi$;

- $(M,(l, u)) \models \phi \wedge \psi$ iff $(M,(l, u)) \models \phi$ and $(M,(l, u)) \models \psi$;

- $(M,(l, u)) \models[i] \phi$ iff $\forall l^{\prime} \in T L, \forall v \in N$, if $\left((l, u),\left(l^{\prime}, v\right)\right) \in R_{i}$, then $\left(M,\left(l^{\prime}, v\right)\right) \models \phi$;

- $(M,(l, u)) \models C \phi$ iff $(M,(l, u))=E^{k} \phi$ for $k=1, \ldots$, where $E^{1} \phi=E \phi, E^{k+1} \phi=E E^{k} \phi$;

- $(M,(l, u)) \models \circ \phi$ iff $(M,(l, u+1)) \models \phi$;

- $(M,(l, u)) \models \phi U \psi$ iff $\exists v \in N$ such that $v \geqslant u$ and $(M,(l, v)) \models \psi$ and $\forall \omega \in N$, if $u \leqslant \omega<v$ then $(M,(l, \omega)) \models \phi$;

- $(M,(l, u)) \models A \phi$ iff for all timelines $l^{\prime}$ of $M$ that are equivalent to $l$ to time $u$, we have $M,\left(l^{\prime}, u\right) \models \phi$.

The semantics we have adopted for the branching quantifier $A$ is called the bundle or the narrow semantics. There is an alternative definition, which is called the broad semantics: $(M,(l, u)) \models A \phi$ if for all timelines $l^{\prime}$ of $M$ and times $u^{\prime}$ with $l(u)=l^{\prime}\left(u^{\prime}\right)$, we have $\left(M,\left(l^{\prime}, u^{\prime}\right)\right) \models \phi$. An $\mathcal{L}$ formula $\phi$ is satisfiable iff there is some $(M,(l, u))$ such that $(M,(l, u)) \models \phi$, and unsatisfiable otherwise. An $\mathcal{L}$ formula $\phi$ is valid in a model $M$ iff $(M,(l, u)) \models \phi$ for every point $(l, u) \in M$. If $\mathcal{C}$ is a class of models, then $\phi$ is $\mathcal{C}$ - valid iff $\phi$ is valid in every model in $\mathcal{C}$. An $\mathcal{L}$ model $M=\left(T L, R_{1}, \ldots, R_{n}, \pi\right)$ is a $K L_{n}$ $\left(B L_{n}\right)$ model iff for all $i \in\{1, \ldots, n\}, R_{i}$ is an equivalence relation (Euclidean, serial and transitive relation).

It is well-known that the following axioms are valid in $B L_{n}$ models: $K:[i] \phi \wedge[i](\phi \supset$ $\psi) \supset[i] \psi D:[i] \phi \supset \neg[i] \neg \phi 4:[i] \phi \supset[i][i] \phi 5: \neg[i] \phi \supset[i] \neg[i] \phi . C: C \phi \supset E \phi \wedge E C \phi$.

It is well-known that the following axioms are valid in $K L_{n}$ models: $K, 4,5, C$ and

$T:[i] \phi \supset \phi$.

There is a graphical interpretation of the semantics $C$ which is useful in the sequel. Fix a model $M$. A point $\left(l^{\prime}, u^{\prime}\right)$ in $M$ is reachable from a point $(l, u)$ if there exists points $\left(l_{0}, u_{0}\right), \ldots,\left(l_{k}, u_{k}\right)$ such that $(l, u)=\left(l_{0}, u_{0}\right),\left(l^{\prime}, u^{\prime}\right)=\left(l_{k}, u_{k}\right)$, and for all $j=0, \ldots, k-1$ there exists $i$ such that $\left(l_{j}, u_{j}\right) R_{i}\left(l_{j+1}, u_{j+1}\right)$. The following result is well known (Halpern et al., 2004).

Lemma 2.1. $(M,(l, u)) \models C \phi$ iff $\left(M,\left(l^{\prime}, u^{\prime}\right)\right) \models \phi$ for all points $\left(l^{\prime}, u^{\prime}\right)$ reachable from $(l, u)$. 


\section{Tait-Style Sequent Calculi}

In this section we introduce Tait-style sequent calculi $K T$ and $B T$ for the branching temporal logics of knowledge and belief.

As usual $p, q, \ldots$ stand for primitive propositions and small Greek letters for arbitrary formulas. Further, the capital Greek letters $\Gamma, \Delta, \Sigma, \ldots$ stand for finite subsets of $\mathcal{L}$ formulas which are called sequents. For any sequents $\Gamma, \Delta$ and formulas $\alpha, \beta$ the sequent $\Gamma \cup \Delta \cup\{\alpha\} \cup\{\beta\}$ is denoted by $\Gamma, \Delta, \alpha, \beta$. Let $\Gamma$ be the sequent $\left\{\alpha_{1}, \ldots, \alpha_{n}\right\}$, we often use the following convenient abbreviations:

- $\vee \Gamma=\left\{\alpha_{1} \vee \ldots \vee \alpha_{n}\right\}$;

- $\neg \Gamma=\left\{\neg \alpha_{1}, \ldots, \neg \alpha_{n}\right\}$;

- $\neg[i] \Gamma=\left\{\neg[i] \alpha_{1}, \ldots, \neg[i] \alpha_{n}\right\}$;

- $[i] \Gamma=\left\{[i] \alpha_{1}, \ldots,[i] \alpha_{n}\right\}$;

- $\circ \Gamma=\left\{\circ \alpha_{1}, \ldots, \circ \alpha_{n}\right\}$;

- $\neg C \Gamma=\left\{\neg C \alpha_{1}, \ldots, \neg C \alpha_{n}\right\}$;

- $A \Gamma=\left\{A \alpha_{1}, \ldots, A \alpha_{n}\right\}$;

- $\neg A \Gamma=\left\{\neg A \alpha_{1}, \ldots, \neg A \alpha_{n}\right\}$.

With the help of de Morgans laws and the law of double negation we push the negation as far as possible, i.e., if $\phi$ is $\alpha \wedge \beta$ then $\neg \phi$ is $\neg \alpha \vee \neg \beta$, if $\phi$ is $\alpha \vee \beta$, then $\neg \phi$ is $\neg \alpha \wedge \neg \beta$, if $\phi$ is $\neg \alpha$, then $\neg \phi$ is $\alpha$.

Let us introduce the Tait-style calculus $K T$ for the branching temporal logic of knowledge. All the rules are represented as schemes.

Axiom of $K T: \Gamma, \alpha, \neg \alpha$

Basic inference rules of $K T$ :

$$
\begin{aligned}
& \frac{\Gamma, \alpha, \beta}{\Gamma, \alpha \vee \beta}(\vee) \quad \frac{\Gamma, \alpha \quad \Gamma, \beta}{\Gamma, \alpha \wedge \beta}(\wedge) \\
& \frac{\neg C \Lambda, \neg[i] \Gamma,[i] \Delta, \alpha}{\neg C \Lambda, \neg[i] \Gamma,[i] \Delta,[i] \alpha, \Sigma}([i]) \quad \frac{\Gamma, \neg \alpha}{\Gamma, \neg[i] \alpha,}(\neg[i])
\end{aligned}
$$

$C$-rules of $K T$ :

$$
\begin{aligned}
& \frac{\Gamma, \neg E \alpha}{\Gamma, \neg C \alpha}(\neg C 1) \quad \frac{\Gamma, \neg E C \alpha}{\Gamma, \neg C \alpha}(\neg C 2) \\
& \frac{\neg \alpha, E \alpha \wedge E \beta}{\neg \alpha, C \beta, \Sigma}\left(I n d_{C}\right)
\end{aligned}
$$

The rules for temporal modalities:

$$
\begin{aligned}
& \frac{\Gamma}{\circ \Gamma, \Sigma}(\circ) \quad \frac{\Gamma, \circ \neg \alpha}{\Gamma, \neg \circ \alpha}(\neg \circ) \\
& \frac{\Gamma, \phi_{2}, \phi_{1} \wedge \circ\left(\phi_{1} U \phi_{2}\right)}{\Gamma, \phi_{1} U \phi_{2}}(U) \quad \frac{\Gamma, \neg \phi_{2} \quad \Gamma, \neg \phi_{1}, \neg \circ\left(\phi_{1} U \phi_{2}\right)}{\Gamma, \neg\left(\phi_{1} U \phi_{2}\right)}(\neg U)
\end{aligned}
$$




$$
\frac{\neg \phi \prime, \neg \psi \wedge \circ \phi^{\prime}}{\neg \phi \prime, \neg(\phi U \psi), \Sigma}\left(\operatorname{Ind}_{U}\right)
$$

The rules for $A$ :

$$
\begin{aligned}
& \frac{\neg A \Gamma, A \Delta, \alpha}{\neg A \Gamma, A \Delta, A \alpha, \Sigma}(A) \quad \frac{\Gamma, \neg \alpha}{\Gamma, \neg A \alpha}(\neg A) \\
& \frac{\Gamma, p}{\Gamma, A p}(A p) \quad \frac{\Gamma, A \circ \alpha}{\Gamma, \circ A \alpha}(\circ A) \quad \frac{\Gamma,[i] \alpha}{\Gamma, A[i] \alpha}(A[i])
\end{aligned}
$$

The sequent calculus $B T$ for the branching temporal logic of belief is obtained from $K T$ by changing the rule $([i])$ by the following rule

$$
\frac{\neg C \Lambda, \neg \Gamma, \neg[i] \Gamma,[i] \Delta, \Theta}{\neg C \Lambda, \neg[i] \Gamma,[i] \Delta,[i] \Theta, \Sigma},
$$

where $\Theta=\emptyset$ or $\Theta=\alpha$ and dropping the rule $(\neg[i])$. We did not introduce any cut rules since we want to distinguish $K T$ and $B T$ with various additional cuts. Hence, we always mention explicitly which cut rules are admitted. Let us introduce the most general cut scheme, the general cut rule.

General cut:

$$
\frac{\Gamma, \alpha \quad \Gamma, \neg \alpha}{\Gamma}(G-c u t) .
$$

In this case the designated formulas $\alpha$ and $\neg \alpha$ are called cut formulas of $(G-c u t)$.

$$
\frac{\Gamma, \alpha \quad \Gamma, \neg \alpha}{\Gamma}(\Pi-c u t)
$$

where $\Pi$ is a subset of formulas closed under the negation, $\alpha \in \Pi$.

Let we have a rule

$$
\frac{\Gamma_{1}}{\Gamma} \text { or } \frac{\Gamma_{1}, \quad \Gamma_{2}}{\Gamma}
$$

of $K T(B T)$. It can be verified that if $\vee \Gamma_{1}$ is $K L_{n}\left(B L_{n}\right)$-valid or $\vee \Gamma_{1}$ and $\vee \Gamma_{2}$ are $K L_{n}\left(B L_{n}\right)$-valid, then $\vee \Gamma$ is $K L_{n}\left(B L_{n}\right)$-valid. So, by induction on the length of the proof it can be showed the following

Theorem 3.1 (soundness). If $K T+(G-c u t) \vdash \Gamma(B T+(G-c u t) \vdash \Gamma)$, then $\vee \Gamma$ is $K L_{n}\left(B L_{n}\right)$-valid.

By the induction on the height of derivations it can be verified the following

Proposition 3.1. The inference rule $\frac{\Gamma}{\Gamma, \alpha}(W)$ is derivable in the calculi $K T$ and $B T$. 


\section{Completeness of $K T$ and $B T$ with a Restricted Cut Rule}

In this section we prove completeness of the Tait-style calculi $K T$ and $B T$ with the cut rule, where the cut formula is from some finite set of formulas. Thus we get proof systems where proof-search becomes decidable (see the next section).

Now we define the Fisher-Ladner closure $F L(\alpha)$ of a formula $\alpha$ of $\mathcal{L}$. $F L(\alpha)$ is defined to be the smallest set such that:

- $\alpha$ belongs to $F L(\alpha)$;

- true $\in F L(\alpha)$;

- if $\neg \beta \in F L(\alpha)$, then $\beta \in F L(\alpha)$;

- if $\beta \vee \gamma \in F L(\alpha)$, then $\beta, \gamma \in F L(\alpha)$;

- if $\beta \wedge \gamma \in F L(\alpha)$, then $\beta, \gamma \in F L(\alpha)$;

- if $[i] \beta \in F L(\alpha)$, then $\beta \in F L(\alpha)$;

- if $C \beta \in F L(\alpha)$, then $E \beta, E C \beta \in F L(\alpha)$;

- if $\circ \beta \in F L(\alpha)$, then $\beta \in F L(\alpha)$;

- if $\beta U \gamma \in F L(\alpha)$, then $\beta, \gamma, \circ(\beta U \gamma) \in F L(\alpha)$;

- if $A \beta \in F L(\alpha)$, then $\beta \in F L(\alpha)$;

- $F L(\alpha)$ is closed under the negation.

We define the closure $F L_{0}(\alpha)$ to be $F L(\alpha) \cup\left\{A\left(\phi_{1} \vee \ldots \vee \phi_{n}\right), \neg A\left(\phi_{1} \vee \ldots \vee\right.\right.$ $\left.\phi_{n}\right) \mid, \phi_{1}, \ldots, \phi_{n}$ are distinct formulas in $\left.F L(\alpha)\right\}$.

As in Fisher et al. (1979) can be verified

Proposition 4.1. For an arbitrary formula $\alpha$ the set $F L(\alpha)$ is finite and contains not more than $c|\alpha|$, where $|\alpha|$ is the length of $\alpha$.

The set $F L_{K}(\alpha)$ is defined to be $F L_{0}(\alpha)$. The set $F L_{B}(\alpha)$ is to be defined $F L_{0}(\alpha) \cup\left\{[i][i] \beta, \neg[i][i] \beta, \mid[i] \beta \in F L_{0}(\alpha), 1 \leqslant i \leqslant n\right\} \cup\{[i] \neg[i] \beta, \neg[i] \neg[i] \beta \| \neg[i] \beta \in$ $\left.F L_{0}(\alpha), 1 \leqslant i \leqslant n\right\}$.

Let $X$ be a finite set of formulas. Then we write $\varphi_{X}$ for the a finite conjunction formulas in $X$.

In the remainder of the paper $W \in\{K, B\}$.

The set $C_{F L_{W}(\alpha)}^{\prime}$ is defined to be the set $\left\{\varphi_{M_{1}} \vee \ldots \vee \varphi_{M_{k}}, \circ\left(\varphi_{M_{1}} \vee \ldots \vee\right.\right.$ $\left.\varphi_{M_{k}}\right),[i]\left(\varphi_{M_{1}} \vee \ldots \vee \varphi_{M_{k}}\right),[i] \neg\left(\varphi_{M_{1}} \vee \ldots \vee \varphi_{M_{k}}\right), \circ A \varphi_{M_{1}} \mid M_{1}, \ldots, M_{k} \subseteq F L_{W}(\alpha), k \geqslant$ 1\} $\cup\left\{A \phi_{M} \mid M \subseteq F L_{W}(\alpha)\right\}$. The closure $C_{F L_{W}(\alpha)}$ is defined to be the set $C_{F L_{W}(\alpha)}^{\prime} \cup$ $\left\{\neg \phi \mid \phi \in C_{F L_{W}(\alpha)}^{\prime}\right\}$. A finite set of $\mathcal{L}$ formulas $\Gamma$ is $C_{F L_{W}(\alpha)}$ - consistent if $W T+$ $\left(C_{F L_{W}(\alpha)}-c u t\right) \forall \neg \Gamma$. We write $\mid \vdash_{W} \Gamma$ if $W T+\left(C_{F L_{W}(\alpha)}-c u t\right) \vdash \Gamma$.

Suppose $C L$ is a finite set if formulas with the property that for all $\phi \in C L$, either $\neg \phi \in C L$ or $\phi$ is of the form $\neg \phi^{\prime}$ and $\phi^{\prime} \in C L$. We define an atom for $W T$ of $C L$ to be a maximal $C_{F L_{W}}(\alpha)$ - consistent subset of $C L$.

First, define a state formula to be a formula $\phi$ such that $\mid \vdash_{W} \neg \phi, A \phi$. We write $X_{S T}$ for the set of states formulas in the set $X$.

Let $\alpha$ be a $C_{F L_{W}(\alpha)}$ - consistent formula. We begin the construction of a $W L_{n}$ model of $\alpha$ by first constructing a pre-model $M_{W}(\alpha)$, which is a structure $<S_{W}, \rightarrow$ 
$, \approx_{A}, R_{1}, \ldots R_{n}>$ consisting of a set $S_{W}$ of states, a binary relation $\rightarrow$ on $S_{W}$, a binary relation $\approx_{A}$ on $S_{W}$ and for each agent $i$ a relation $R_{i}$ on $S_{W}$, which is an equivalence (Euclidean, serial, transitive) relation for $W=K(W=B)$.

The set $S_{W}$ consists of all atoms of $F L_{W}(\alpha)$. The relation $\rightarrow$ is defined so that $X \rightarrow Y$ iff not $\mid \vdash_{W} \neg \varphi_{X}, \neg \circ \varphi_{Y}$. The relation $R_{i}$ on $S_{B}$ is defined so that $(X, Y) \in R_{i}$ iff $\{\phi \mid[i] \phi \in X\} \subseteq Y$. The relation $R_{i}$ on $S_{K}$ is defined so that $(X, Y) \in R_{i}$ iff $\{\phi \mid[i] \phi \in$ $X\}=\{\phi \mid[i] \phi \in Y\}$. The relation $\approx_{A}$ is defined so that $X \approx_{A} Y$ iff $X_{S T}=Y_{S T}$. As in Alberucci (2002) it can be proved

Lemma 4.1. If $X \subseteq F L_{W}(\alpha)$, and $X$ is $C_{F L_{W}(\alpha)}$ - consistent, then there exists an atom $Y$ of $F L_{W}(\alpha)$ such that $X \subseteq Y$.

Using the definition of an atom we can verify the following

Proposition 4.2. 1) If $X$ is an atom of $F L_{W}(\alpha), \phi \in F L_{W}(\alpha)$ and $\mid \vdash_{W} \neg X, \phi$, then $\phi \in X$;

2) If $X$ is an atom of $F L_{W}(\alpha), \phi \in F L_{W}(\alpha)$, then $\phi \notin X$ iff $\neg \phi \in X$;

Using the definition of a state formula we can prove the following

PROPOSITION 4.3. 1) If $\phi$ is a state formula, then $\neg \phi$ is a state formula;

2) If $\phi$ is a primitive proposition, or $\phi$ is of the form $[i] \phi_{1}, A \phi_{1}$, then $\phi$ is a state formula;

3) If $s$ is a state in $S_{W}, \phi \in s$ and $\phi$ is a state formula, then $\mid \vdash_{W} \neg \phi_{s}, A \phi$.

Let $S_{B}$ be a set of states in the pre-model $M_{B}(\alpha)$ and $X, Y, Z$ be states from $S_{B}$.

Lemma 4.2. - For each $X \in S_{B}$ there exists $Y \in S_{B}$ such that $(X, Y) \in R_{i}$.

- if $[i] \beta \in X$ and $(X, Y) \in R_{i}$, then $[i] \beta \in Y$;

- if $(X, Y) \in R_{i}$ and $(X, Z) \in R_{i}$ and $[i] \beta \in Y$, then $\beta \in Z$.

From Lemma 4.2 it follows that the relation $R_{i}$ in $M_{B}(\alpha)$ is a serial, transitive and Euclidean relation for each $i \in\{1, \ldots, n\}$.

In the remainder of the paper "state" means a state in $S_{W}$.

Let $U$ be a set of states. We write $\varphi_{U}$ for disjunction of the formulas $\varphi_{u}$ for $u \in U$.

Define a $\rightarrow$ - sequence of states to be a (finite or infinite) sequence $s_{0}, s_{1}, \ldots$ such that $s_{0} \rightarrow s_{1} \rightarrow \ldots$ (in particular, $\rightarrow-$ sequence may consist of one element).

As in Sakalauskaitè (2004), Sakalauskaite (2006) it can be verified.

Lemma 4.3. a) if $\circ \phi \in F L_{W}(\alpha)$, then for all states $t$ such that $s \rightarrow t$ we have $\circ \phi \in$ siff $\phi \in t$;

b) if $[i] \phi \in F L_{W}(\alpha)$, then $\neg[i] \phi \in s$ iff there is some state $t$ such that $s R_{i} t$ and $\neg \phi \in t$; 
c) if $\phi_{1} U \phi_{2} \in F L_{W}(\alpha)$, then $\phi_{1} U \phi_{2} \in s$ iff there exists $a \rightarrow$-sequence $s=s_{0} \rightarrow s_{1} \rightarrow \ldots \rightarrow s_{n}$, where $n \geqslant 0$ such that $\phi_{2} \in s_{n}$ and $\phi_{1} \in s_{k}$ for all $k<n$.

d) If $C \phi \in F L_{W}(\alpha)$, then $\neg C \phi \in s$ iff there is a state t reachable from s through the relations $R_{i}, 1 \leqslant i \leqslant n$, such that $\neg \phi \in t$.

Lemma 4.4. If $A \phi \in F L_{W}(\alpha)$ and $A \phi \in s$, then for all states $t$ such that $s \approx_{A} t$, we have $\phi \in t$.

Proof. If $A \phi \in s$ and $s \approx_{A} t$, then $A \phi \in t$. Hence $\neg \phi \notin t$ by $(\neg A)$. So $\phi \in t$.

Lemma 4.5. If $A \phi \in F L_{W}(\alpha)$ and $\neg A \phi \in s$, then there exists a state $t$ such that $s \approx_{A} t$ and $\neg \phi \in t$.

Proof. Suppose $A \phi \in F L_{W}(\alpha)$ and $\neg A \phi \in s$, i.e., $\mid \vdash_{W} \neg \varphi_{s}, \neg A \phi$. We show by contraposition that $s_{S T} \cup\{\neg \phi\}$ is $C_{F L_{W}(\alpha)}$ - consistent. Let $s_{S T} \cup\{\neg \phi\}$ is $C_{F L_{W}(\alpha)}$ inconsistent, i.e., $\mid \vdash_{W} \neg \varphi_{s_{S T}}, \phi$. So, by the rules $(A),(\neg A) \mid \vdash_{W} \neg A\left\{\phi_{1}, \ldots, \phi_{k}\right\}, A \phi$, where $s_{S T}=\left\{\phi_{1}, \ldots, \phi_{k}\right\}$. But $\mid \vdash_{W} \neg \varphi_{S_{S T}}, A \phi_{i}, 1 \leqslant i \leqslant k$. So by the appropriate cuts we get $\mid \vdash_{W} \neg \varphi_{s_{S T}}, A \phi$. Since $\mid \vdash_{W} \neg \varphi_{s}, \phi_{i}$ we derive $\mid \vdash_{W} \neg \varphi_{s}, A \phi$. Contradiction. Thus $s_{S T} \cup\{\neg \phi\}$ is $C_{F L_{W}(\alpha)}$ - consistent, and by Lemma 4.1 there exists an atom $t$ of $F L_{W}(\alpha)$ such that $s_{S T} \cup\{\neg \phi\} \subseteq t$. Clearly, $\neg \phi \in t$. Moreover, $s \approx_{A} t$. This is because $t$ contains $s_{S T}$, which in turn contains either $\phi$ or $\neg \phi$ for every state formula $\phi \in F L_{W}(\alpha)$.

$$
\text { Let } \exists={ }_{d f} \neg A \neg \text {. }
$$

Lemma 4.6. Suppose that $s, t$ are states such that $s \approx_{A}$ t. Then $\mid \vdash_{W} \neg \varphi_{s}, \exists \varphi_{t}$.

Proof. From $s \approx_{A} t$ we have $s_{S T}=t_{S T}$ (1). Write $t=\left\{\phi_{1}, \ldots, \phi_{k}, \psi_{1}, \ldots, \psi_{l}\right\}$, where $t \cap F L(\alpha)=\left\{\phi_{1}, \ldots, \phi_{k}\right\}$. Note that all remaining formulas $\psi_{j}$ are of he form $A \phi$ or $[i] \phi$, or negations of these forms, hence state formulas. From (1) it follows $\mid \vdash_{W}$ $\neg \varphi_{s}, A \wedge_{j=1}^{l} \psi_{j}$.

Let $\beta=A \vee_{j=1}^{k} \neg \phi_{j}$. By construction, $\beta \in F L_{0}(\alpha)$. Hence $\mid \vdash_{W} \neg \varphi_{t}, \beta$ or $\mid \vdash_{W}$ $\neg \varphi_{t}, \neg \beta$. Since $\mid \vdash_{W} \neg \beta, \vee_{j=1}^{k} \neg \phi_{j}$ by the rule $(\neg A)$ and $t$ is $C_{F L_{W}(\alpha)}$ - consistent we cannot have $\mid \vdash_{W} \neg \varphi_{t}, \beta$. It follows $\neg \beta \in t$. Hence $\neg \beta \in s$, since $\neg \beta$ is a state formula. Thus $\mid \vdash_{W} \neg \varphi_{s}, \neg \beta$. Hence $\mid \vdash_{W} \neg \varphi_{s}, \exists \wedge_{j=1}^{k} \phi_{j}$. We have

$$
\mid \vdash_{W} w, \neg \exists \delta, \neg A \gamma, \exists(\gamma \wedge \delta)
$$

and

$$
\frac{\left|\vdash_{W}, w, \exists \delta \quad\right| \vdash_{W} w, \neg \exists \delta, \neg A \gamma, \exists(\gamma \wedge \delta)}{\mid \vdash_{W} w, \neg A \gamma, \exists(\gamma \wedge \delta)} .
$$

With $\mid \vdash_{W} w, \exists \delta$ and $\mid \vdash_{W} w, A \gamma$ we get $\mid \vdash_{W} w, \exists(\gamma \wedge \delta)$. Take $w=\neg \varphi_{s}, \gamma=$ $\wedge_{j=1}^{l} \psi_{j}, \delta=\wedge_{j=1}^{k} \phi_{j}$. Lemma follows. 
We say that an infinite $\rightarrow$-sequence of states $\left(s_{0}, s_{1}, \ldots\right)$ is acceptable if for all $n \geqslant 0$, if $\phi_{1} U \phi_{2} \in s_{n}$, then there exists $m \geqslant n$ such that $\phi_{2} \in s_{m}$ and $\phi_{1} \in s_{k}$ for all $n \leqslant k<m$.

Similar as in Halpern et al. (2004), Sakalauskaitè (2004) using c) of Lemma 4.3 we can verify

Lemma 4.7. Every finite $\rightarrow$-sequence of states can be extended to an infinite acceptable sequence.

The following lemma concerning the branching operator will be useful for our constructions.

Below $s, s^{\prime}, t^{\prime}$ are states from the set $S_{W}$ from the pre-model $M_{W}(\alpha)$.

Lemma 4.8. Suppose that $s, s^{\prime}, t^{\prime}$ are states such that $s \rightarrow s^{\prime}$ and $s^{\prime} \approx_{A} t^{\prime}$. Then there exists a state $t$ such that $t \rightarrow t^{\prime}$ and $s \approx_{A} t$.

Proof. The proof follows the lines of the corresponding Lemma in van der Meyden et al.(2003). We proceed by contradiction. Suppose that $s \rightarrow s^{\prime}$ and $s^{\prime} \approx_{A} t^{\prime}$ and there does not exist a state $t$ such that $t \rightarrow t^{\prime}$ and $s \approx_{A} t$. Then for all states $t$, if $s \approx_{A} t$ then $\mid \vdash_{W} \neg \varphi_{t}, \neg \circ \varphi_{t^{\prime}}$ (by the definition of $\rightarrow$ ). By the definition of $\approx_{A}$ it follows that $\mid \vdash_{W} \neg \varphi_{s_{S T}}, \vee_{t: s \approx_{A}} \varphi_{t}$. So, $\mid \vdash_{W} \neg \varphi_{s_{S T}}, \neg \circ \varphi_{t^{\prime}}$. Since $\mid \vdash_{W} \circ \varphi_{t^{\prime}}, \circ \neg \varphi_{t^{\prime}}$ (by the rule (०)) we get $\mid \vdash_{W} \neg \varphi_{s_{S T}}, \circ \neg \varphi_{t^{\prime}}$. By the rules $(\neg A)$ and $(A)$ we derive $\mid \vdash_{W} \neg A \varphi_{s_{S T}}, A$ 。 $\neg \varphi_{t^{\prime}}$. Using the rule $(\circ A)$ we get $\mid \vdash_{W} \neg A \varphi_{s_{S T}}, \circ A \neg \varphi_{t^{\prime}}$. Since $\mid \vdash_{W} \neg \varphi_{s}, A \phi$, where $\phi \in s_{S T}$, by the appropriate cuts, we have $\mid \vdash_{W} \neg \varphi_{s}, \circ A \neg \varphi_{t^{\prime}}(1)$.

Since $s \rightarrow s^{\prime}$, we have not $\mid \vdash_{W} \neg \varphi_{s}, \neg \circ \varphi_{s^{\prime}}$. Since

$$
\frac{\neg \varphi_{s^{\prime}}, \neg A \neg \varphi_{t^{\prime}}}{\neg \circ \varphi_{s^{\prime}}, \neg \circ A \neg \varphi_{t^{\prime}}},
$$

using (1), by the appropriate cut we get that not $\mid \vdash_{W} \neg \varphi_{s^{\prime}}, \neg A \neg \varphi_{t^{\prime}}$. By Lemma 4.6 and the assumption that $s^{\prime} \approx_{A} t^{\prime}$, we have $\mid \vdash_{W} \neg \varphi_{s^{\prime}}, \exists \varphi_{t^{\prime}}$. This is a contradiction.

A canonical model for $\alpha$ is a tuple $\left(\mathcal{R}, R_{1}^{\prime}, \ldots, R_{n}^{\prime}, \pi\right)$, where $\mathcal{R}$ is a set of all sequences $s_{S T}^{0}, s_{S T}^{1}, \ldots$, where $s^{0}, s^{1}, \ldots$ is an acceptable sequence of states in $M_{W}(\alpha)$; Let $(r, n)$ and $\left(r^{\prime}, n^{\prime}\right)$ be points in $\mathcal{R} \times N$. So $r(n)=s_{S T}, r^{\prime}\left(n^{\prime}\right)=t_{S T}$ for some states $s, t$ in $M_{W}(\alpha)$. Then $R_{i}^{\prime}$ is a binary relation on points in $\mathcal{R} \times N$ such that $(r, n) R_{i}^{\prime}\left(r^{\prime}, n^{\prime}\right)$ iff $s R_{i} t$. Let $r(n)=s_{S T}$. Then $\pi(r, n)(p)=T$ iff $p \in s$. The following theorem gives a sufficient condition for a formula in the Fisher-Ladner closure to hold at a point in the canonical model. Let $s^{0}, s^{1}, \ldots$ be an acceptable sequence of states and $r$ be a timeline obtained from that sequence, i.e., $r(n)=s_{S T}^{n}$. We call the state $s^{n}$ corresponding to the point $(r, n)$.

Theorem 4.1. Let $W \in\{K, B\}$. If $\mathcal{I}$ is the canonical model for $\alpha, \phi$ is in $F L_{W}(\alpha)$, then $(\mathcal{I},(r, n)) \models \phi$ if and only if $\phi \in s$, where $s$ is the state, corresponding to the point $(r, n)$. 
Proof. We proceed by induction on the complexity of $\phi$. The cases for $\phi$ of the form $\neg \phi_{1}, \phi_{1} \vee \phi_{2}, \phi_{1} \wedge \phi_{2}$ are trivial. The cases for $\phi$ of the form $\circ \phi_{1}, \phi_{1} U \phi_{2},[i] \phi_{1}, C \phi_{1}$ are proved as in Sakalauskaite (2006) using Lemma 4.3 and Lemma 4.7. We prove the case $\phi=A \phi_{1}$.

We prove the "only if" part. Suppose $A \phi_{1} \in s$. Then by Lemma 4.4, we have $\phi_{1} \in t$ for all states $t$ such that $s \approx_{A} t$. Then by the induction hypothesis we get that $\left.\left(\mathcal{I},\left(r \prime, n^{\prime}\right)\right) \models \phi_{1}\right)$,where $t$ is the corresponding state to the point $(r \prime, n \prime)$. Let $r^{\prime}$ be a timeline such that $r$ is equivalent to $r$ to time $n$. Then we have $s \approx_{A} t /$, where $t /$ is a state corresponding to the point $(r \prime, n)$. Thus, $(\mathcal{I},(r \prime, n)) \models \phi_{1}$. Thus, $(\mathcal{I},(r, n)) \models \phi$.

Conversely, suppose that $\neg A \phi_{1} \in s$, where $s$ corresponds to the point $(r, n)$. Thus we have an acceptable sequence $\Sigma=s_{0}, \ldots, s_{n}=s, \ldots$ such that $r$ is obtained from $\Sigma$. By Lemma 4.5, there exists a state $t$ such that $\neg \phi_{1} \in t$ and $s \approx_{A} t$. By repeated application of Lemma 4.8, there exist states $t_{0}, \ldots, t_{n}$ such that $t=t_{n}, s_{i} \approx_{A} t_{i}$ for $0 \leqslant i \leqslant n$ and $t_{0} \rightarrow, \ldots \rightarrow t_{n}$. By Lemma 4.7, we may extend this sequence to an acceptable sequence $t_{0}, \ldots, t_{n}, \ldots$, Let $r /$ be the timeline, obtained from this sequence. By the definition of timelines it follows that $r$ is equivalent to $r$ to time $n$. By the induction hypothesis we have $(\mathcal{I},(r \prime, n)) \models \neg \phi_{1}$, hence $(\mathcal{I},(r, n)) \models \neg A \phi_{1}$.

COROLlary 4.1. If $\mathcal{I}$ is the canonical model for $\alpha,(r, n)$ is a point of $\mathcal{I}$ such that $\alpha \in s$, where $s$ is the corresponding state to the point $(r, n)$, then $(\mathcal{I},(r, n)) \models \alpha$.

Let $W \in\{K, B\}$. Assume that $\alpha$ is $C_{F L_{W}(\alpha)}$ - consistent formula. Let $s$ be a state in $S_{W}$ such that $\alpha \in s$. Such a state must exist as follows by Lemma 4.1. By Lemma 4.7 there exists an acceptable sequence $s_{0}, s_{1}, \ldots$ with $s=s_{0}$. Let $r$ be the corresponding timeline in the canonical model $\mathcal{I}$ for $\alpha$. Corollary 4.1 implies that $(\mathcal{I},(r, 0)) \models \alpha$. This establishes the following completeness theorem for the calculi $K T+\left(C_{F L_{K}(\alpha)}-c u t\right)$ and $B T+\left(C_{F L_{B}(\alpha)}-c u t\right)$

Theorem 4.2 (completeness). Let $\alpha$ be a $K L_{n}\left(B L_{n}\right)$ - valid formula. Then $K T+$ $\left(C_{F L_{K}(\alpha)}-c u t\right) \vdash \alpha\left(B T+\left(C_{F L_{B}(\alpha)}-c u t\right) \vdash \alpha\right)$.

\section{Decidability of Provability}

In this section we define the sequent calculi $K T^{\prime}, B T^{\prime}$ obtained from the calculi $K T, B T$ by replacing the induction rules $\left(I n d_{U}\right),\left(\operatorname{Ind} d_{C}\right)$ by the slightly modified induction rules. Then we prove the decidability of provability in these sequent calculi together with the restricted cut rule.

We define the rules $\left(\operatorname{Ind} d_{C}^{\prime}\right)$ and $\left(\operatorname{Ind}_{U}^{\prime}\right)$ as follows:

$$
\frac{\neg \phi^{\prime}, \neg \psi \wedge \circ \phi^{\prime}}{\neg \phi^{\prime}, \neg\left(\text { trueU } \neg \phi^{\prime}\right) \wedge \neg(\phi U \psi)}\left(\operatorname{Ind}_{U}^{\prime}\right) \quad \frac{\neg \phi, E \phi \wedge E \psi}{\neg \phi, C \phi \wedge C \psi}\left(\operatorname{Ind}_{C}^{\prime}\right)
$$

These rules are derivable in the calculi $K T+(G-c u t), B T+(G-c u t)$. So they are sound. 
Let $K T^{\prime}, B T^{\prime}$ be the calculi obtained from the calculi $K T, B T$ by replacing the rules $\left(I n d_{C}\right),\left(I n d_{U}\right)$ by the rules $\left(I n d_{C}^{\prime}\right),\left(I n d_{U}^{\prime}\right)$. The rules $\left(I n d_{C}\right),\left(I n d_{U}\right)$ are derivable in the calculi $K T^{\prime}, B T^{\prime}$ if we have cut rules with additional cut formulas. In the case of the rule $\left(I n d_{U}\right)$ these cut formulas are of the form $\neg\left(\operatorname{true} U \neg \phi^{\prime}\right) \wedge \neg(\phi U \psi)$. In the case of the rule $\left(I n d_{C}\right)$ these cut formulas are of the form $C \phi \wedge C \psi$.

We extend the closure set of cut formulas $C_{F L_{W}(\alpha)}$ to the set $C_{F L_{W}(\alpha)}^{\prime \prime}$ by adding the set of additional cut formulas $\Phi_{W}=\left\{\neg\left(\right.\right.$ true $\left.U \neg \psi^{\prime}\right) \wedge \neg(\phi U \psi)$, $\left(\right.$ true $\left.\left.U \neg \psi^{\prime}\right) \vee(\phi U \psi) \mid \phi U \psi \in F L_{W}(\alpha), \psi^{\prime} \in C_{F L_{W}(\alpha)}\right\} \cup\{C \phi \wedge C \psi, \neg C \phi \vee$ $\left.\neg C \psi \mid C \phi \in F L_{W}(\alpha), \psi \in C_{F L_{W}}(\alpha)\right\}$.

We define the set of formulas $\bar{\Phi}_{W}$ such that:

- they are obtained from formulas in $C_{F L_{W}}(\alpha) \cup \Phi_{W}$ using the inference rules of $W T^{\prime}+\left(C_{F L_{W}(\alpha)}^{\prime \prime}-\right.$ cut $)$ upwards;

- formulas from $\bar{\Phi}_{W}$ are not included in $C_{F L_{W}}(\alpha) \cup \Phi_{W}$.

$\bar{\Phi}_{W}$ is defined as follows: $\left\{\neg\left(\right.\right.$ true $\left.U \neg \psi^{\prime}\right), \neg \circ\left(\right.$ true $\left.U \neg \psi^{\prime}\right), \circ \neg\left(\right.$ true $\left.U \neg \psi^{\prime}\right)$, $\neg \psi \wedge \circ \psi^{\prime}, \circ \psi^{\prime}$, true $U \neg \psi^{\prime}$, true $\wedge \circ\left(\right.$ true $\left.U \neg \psi^{\prime}\right), \circ\left(\right.$ true $\left.U \neg \psi^{\prime}\right) \mid \phi U \psi \in F L_{W}(\alpha)$, $\left.\psi^{\prime} \in C_{F L_{W}}(\alpha)\right\} \cup\{C \psi, E \phi \wedge E \psi, E \psi,[1] \psi, \ldots,[n] \psi, \neg C \psi, \neg E \psi, \neg E C \psi$, $\left.\neg[1] \psi, \ldots, \neg[n] \psi, \neg[1] C \psi, \ldots, \neg[n] C \psi, \mid C \phi \in F L_{W}(\alpha), \psi \in C_{F L_{W}}(\alpha)\right\} \cup\{E \phi \wedge$ $\left.E \psi \mid C \phi \wedge C \psi \in C_{F L_{W}}(\alpha)\right\} \cup\left\{A \circ \phi, \mid \circ A \phi \in C_{F L_{W}(\alpha)}\right\} \cup\left\{\circ \neg \phi \mid \neg \circ \phi \in C_{F L_{W}(\alpha)}\right\}$.

Let $\bar{C}_{F L_{W}(\alpha)}=C_{F L_{W}(\alpha)}^{\prime \prime} \cup \bar{\Phi}_{W}$. Using Proposition 4.1 we can verify that the set $\bar{C}_{F L_{W}(\alpha)}$ is finite.

We can verify

PROPOSITION 5.1. Let

$$
\frac{\Gamma_{1}}{\Gamma} \quad \text { or } \frac{\Gamma_{1} \quad \Gamma_{2}}{\Gamma}
$$

be an inference rule in the calculus $W T^{\prime}+\left(C_{F L_{W}}^{\prime \prime}(\alpha)-c u t\right)$. If $\Gamma \subseteq \bar{C}_{F L_{W}(\alpha)}$ then $\Gamma_{1}, \Gamma_{2} \subseteq \bar{C}_{F L_{W}(\alpha)}$.

From the proposition above it follows that the number of distinct sequents in any branch generated by the inference rules upward from $\alpha$ is finite.

Now we describe the following decision algorithm to check if a formula $\alpha$ is provable in the calculus $W T^{\prime}+\left(C_{F L_{W}(\alpha)}^{\prime \prime}-c u t\right)$ or not, where $W \in\{K, B\}$.

Algorithm. Try to construct the proof from the end sequent upward. It is clear that for the last inference there are finitely many possibilities. We will repeat this again for the sequents which are obtained in this way. When we reach a sequent which doesn't produce any new sequent on the considered branch the construction of this branch stops and we check whether this leaf it is an axiom. Since the number of distinct sequents in any such branch is finite the construction of each branch stops. Note that the number of trees generated in such a way is finite. If in this set of trees we get the tree such that all the leafs are axioms the formula $\alpha$ is provable. Otherwise, we fail. 


\section{References}

Alberucci, L. (2002). The modal $\mu$-calculus and the logic of common knowledge. PhD Thesis, Institut für Informatik und angewandte Mathematik, Universität Bern.

Catach, L. (1988). Normal multimodal logics. In Proccedings of the Seventh National Conference in Artificial Intelligence. St. Paul, MN. pp. 491-495.

Dixon, C., M. Fisher, M. Wooldridge (1998). Resolution for temporal logics of knowledge. J. Logic Computat., 8(3), 345-372.

Fagin, R., J.Y. Halpern, Y. Moses and M.Y. Vardi (1995). Resoning about Knowledge. MIT Press.

Fisher, M.J., R.E. Ladner (1979). Propositional dynamic logic of regular programs. J. Computer and System Sciences, 18, 194-211.

Halpern, J.Y., Y. Moses (1992). A guide to completeness and complexity for modal logics of knowledge and belief. Artificial Intelligence, 54, 319-379.

Halpern, J.Y., R. van der Meyden, M.Y. Vardi (2004). Complete axiomatizations for reasoning about knowledge and time. SIAM J. on Computing, 33, 674-703.

van der Meyden, R., K.S. Wong (2003). Complete axiomatizations for reasoning about knowledge and branching time. Studia Logica, 75, 93-123.

Sakalauskaitè, J. (2004). Sequent calculi with analytic cut for logics of time and knowledge with perfect recall. Lith. Math. J., 44(2), 209-224.

Sakalauskaite, J. (2006). Sequent calculi for temporal logics of common knowledge and belief. Informatica, 17(1), 85-94.

Stirling, C. (1992). Modal and temporal logics. In S. Abramsky et al. (Eds.), Handbook of Logic in Computer Science, vol. 2, Clarendon Press, Oxford. pp. 477-563.

Wooldridge, M. (1992). The logical modelling of computational multi-agent systems. PhD Thesis, Department of Computation, UMIST, Manchester, UK, October.

Wooldridge, M., C. Dixon, M. Fisher (1998). A tableau-based proof method for temporal logics of knowledge and belief. Journal of Applied Non-Classical Logics, 8(3), 225-258.

J. Sakalauskaitė received her PhD degree from Institute of Mathematics of Sankt Peterburg branch, Russia in 1986. She is a senior researcher of Institute of Mathematics and Informatics of Vilnius. Her research interests include proof theory of non-classical logics such as modal, temporal, dynamic, agent ones.

\section{Sekvenciju skaičiavimas skaidaus laiko žiniu ir tikejjimo logikoms}

\section{Jūratė SAKALAUSKAITÉ}

Nagrinèjamos skaidaus laiko žinių ir tikèjimo logikos. Skaidaus laiko operatoriaus "visiems keliams" semantika yra apribota. Šioms logikoms pateikti sekvencijų skaičiavimai su apribota pjūvio taisykle. Irodytas šių skaičiavimų korektiškumas, pilnumas ir išsprendžiamumas. 